\title{
Role of 2d Speckled Tracking Echocardiography in Assessment of in Hospital and One Year Outcome of Patients with Acute Myocardial Infarction Versus Chronic Coronary Artery Disease
}

\author{
K.E.ElRabaat, A.I.Attia, H.H.Ebaid and G.G.Shaker \\ Cardiology Dept., Faculty of Medicine, Benha Univ., Benha, Egypt \\ E-Mail: George@gmail.com
}

\begin{abstract}
Two-dimensional speckle strain (2D STE) echocardiography can aid in the prognosis of acute myocardial infarction (AMI) and chronic coronary artery disease (CAD). the aim of the present study was to determine the possibility of using of two-dimensional speckle tracking echocardiography to predict cardiac events comparing of patients with acute myocardial infarction versus chronic coronary artery disease. this was a prospective study includes two hundred patients (20-70 years old) in two groups were selected from national heart institute and El Sahel hospitals first group one hundred of patients with a diagnosis of first attack of acute myocardial infarction (STEMI or NSTEMI) and the other group one hundred of patients with known chronic CAD who presented for clinical checkup at the outpatient department. The duration of the study ranged from 6-12 months. as regard strain parameters in patients with first acute MI; there was high significant difference between cases with cardiac event and those without as regard GLS epi \%, GLS total wall, \% and GCS endo, \%, also there was significant difference between cases with cardiac event and those without as regard GLS endo, (\%),GCS epi,\% and GCS total wall, $\%$., and there was highly statistical difference between chronic coronary heart diseases cases with cardiac event and those without as regard conventional echocardiography and strain parameters. Global strain parameters by 2D STE may be useful for the prediction of cardiac events in patients with CAD but add no supplemental information to baseline characteristic and ejection fraction in patients with AMI.
\end{abstract}

Keywords: Acute Myocardial Infarction, Cardiac Event, Coronary Artery Disease, Echocardiography.

\section{Introduction}

Myocardial remodeling occurs prior to the development of cardiac disease; consequently, international guidelines have underlined the importance of detecting subclinical left ventricular (LV) dysfunction [1].

Furthermore, LV systolic function is recognized as a major predictor of outcome after acute myocardial infarction (AMI).2 As a result, echocardiography following AMI is implemented as a routine procedure for risk stratification. Frequently used measurements for echocardiographic quantification of global and regional LV systolic function include LV ejection fraction (LVEF) and wall motion score index (WMSI) [2] .

However, in recent years, more novel echocardiographic techniques, such as tissue Doppler imaging and 2-dimensional strain echocardiography (2D STE), have been shown to be superior to prognostic measures compared to the LVEF and WMSI [3] 2D STE has been recognized in a range of studies to provide important prognostic information in patients with coronary artery disease (CAD) 6,7 and AMI [4].

However, the majority of studies that have evaluated the usefulness of novel myocardial deformation measures in patients with CAD and AMI have mainly focused on the prognostic use of only global longitudinal deformation parameters and rarely evaluated layer specific data (endocardial and epicardial myocardium) [5] To date, it is still unclear whether there is a difference in the sensitivity or characteristics of 2D STE parameters for the prediction of prognosis in patients with AMI or CAD.

\section{Subjects and methods}

This prospective study includes two hundred patients (20-70 years old) in two groups had been selected from national heart institute and El Sahel hospitals first group one hundred of patients with a diagnosis of first attack of acute myocardial infarction (STEMI or NSTEMI) and the other group one hundred of patients with known chronic CAD who presented for clinical checkup at the outpatient department.

\section{Inclusion criteria \\ First group}

A diagnosis of acute myocardial infarction had been made on the basis of: Persistent chest discomfort or other symptoms suggestive of ischaemia. ST segment elevation in at least two contiguous leads as STEMI, in contrast patients without ST segment elevation may have ST or T wave changes. Elevation of creatine Kinase enzyme and its myocardial band (MB) fraction to more than twice the upper limit of normal and elevation of cardiac troponin values with at least one value above the $99^{\text {th }}$ percentile upper reference limit with necrosis in a clinical setting consistent with myocardial ischaemia. AMI patients must be successfully revascularized in all perfusion beds.

\section{Second group}

Patients with known CAD who presented for clinical checkup at the outpatient department and underwent echocardiography during the same period and they were on optimal medical treatment for at least 3 months and coronary revascularized according to current guidelines [6] prior to being included in the study.

\section{Exclusion criteria}

In both groups, patients with relevant valvular heart disease were excluded. Because of the emergency valuation of the AMI group, we choose the following procedure: after providing information about the study process to the patient by the study physician, an 
independent physician was asked to assess the patient by checking all inclusion and exclusion criteria. Only upon acceptance of the patient by the independent physician is the patient included in the study. After the procedure, the patient will be questioned again as to whether he or she is still willing to participate in the study after 48 hours and is able to revoke his or her consent indefinitely. All included patients provided written informed consent.

\section{Method}

All the patients will be subjected to:

1- History taking: A careful history had been taken from the patient especially the chest pain analysis and the associated symptoms, past history of any chronic diseases, ex: diabetes \& hypertension any treatments the patient receive and whether the patient smokes or not.

2- General examination: Including the examination of head and face, skin, extremities, chest and abdominal examination, JVP, BP, Arterial pulse and temp.

3- Local examination: Including Inspection, palpation, percussion and auscultation .

4- ECG: The ECG had been done on admission and will be studied as follows: ST segment deviation from isoelectric line which had been defined as the level of the proceeding TP segment had been measured $80 \mathrm{~ms}$ after the $\mathrm{J}$ point to the nearest $0.5 \mathrm{~mm}$.

5- Lab findings: Plasma levels of creatine kinase \& creatine kinase $-\mathrm{MB}$ fraction will be assessed every 8 hours in the first 24 hours. -Troponin had been done on admission [1].

6- Echocardiography: Left ventricular parasternal shortaxis views at basal, midventricular, and apical levels were acquired, as well as 2-, 3- and 4-chamber views. A 18-segment model with 6 segments at the basal, midventricular, and apical short-axis level was used to divide the LV. Wall motion was assessed by visual interpretation for each LV segment as normokinetic, mildly hypokinetic, severely hypokinetic, akinetic, or dyskinetic. WMSI was calculated for each patient as the average of the analyzed segmental values. LVEF and end-systolic and end-diastolic volumes were assessed by biplane Simpson's method using manual tracing of digital images. All measurements were performed in 3 consecutive heart beats and were averaged. In the AMI group, echocardiographic examinations were performed within 48 hours of coronary angiography. All segmental values were averaged to global data. All echocardiographic data were analyzed by 2 blinded experienced cardiologists. To avoid confusion, we expressed all strain data as absolute values
7- Coronary angiography: Using standard techniques, $\mathrm{x}$ ray coronary angiography was performed. The severity of coronary stenosis was determined quantitatively using Philips softwere. All decisions on coronary interventions and subsequent clinical care were based on the discretion of the treating cardiologist. Finally coronary angiographic findings will be correlated with the ECG findings in both groups.

8- Clinical follow-up: Clinical follow-up was performed every 3 months for one year based on telephone contact.

\section{Statistical analysis}

Data collected throughout history, basic clinical examination, laboratory investigations and outcome measures coded, entered and analyzed using Microsoft Excel software. Data were then imported into Statistical Package for the Social Sciences (SPSS version 20.0) (Statistical Package for the Social Sciences) software for analysis.

\section{Results}

Conventional echocardiographic parameters: -

There is no significant difference between the studied groups as regard LVESV; also there is no significant difference between the studied groups as regard LVEDV and EF. LVEDV: The Mean LVEDV was [110.7 \pm 11$]$ lv for the CAD group and [109.4 \pm 24.04$] \mathrm{lv}$ for the AMI group, $(\mathrm{P}$ value $=0.134)$. LVESV: The Mean LVESV was $[41.8 \pm 5.6] \mathrm{lv}$ for the CAD group and $[40.3 \pm 9.6] \mathrm{lv}$ for the AMI, $(\mathrm{P}$ value $=0.314)$. EF $(\%)$ : The Mean EF $(\%)$ : was $[67.56 \pm 3.77] \mathrm{cm}$ for the CAD group and $[67.3 \pm 3.34] \mathrm{cm}$ for the AMI, $(\mathrm{P}$ value $=0.801)$.

\section{Strain parameters}

There was no statistical significant difference between the two studied groups as regard strain parameters Table [1].

This table shows that there is high significant difference between cases with cardiac event and those without as regard LVEDV (lv) and EF\%, also there is significant difference between cases with cardiac event and those without as regard LVESV (lv) Table [2].

This table shows that there is high significant difference between cases with cardiac event and those without as regard GLS epi,\%, GLS total wall, \% and GCS endo, $\%$, also there is significant difference between cases with cardiac event and those without as regard GLS endo, (\%),GCS epi,\% and GCS total wall,\%. Table [3].

This table shows that there is significant difference between cases with cardiac event and those without as regard LVEDV (lv), EF\%, and LVESV (lv) Table [4].

Table (1) Comparison between the two studied groups as regard conventional echocardiography parameters.

\begin{tabular}{lcccc}
\hline Variable & AMI & CAD & T test & P value \\
& $\mathbf{N = 1 0 0}$ & $\mathbf{N = 1 0 0}$ & & \\
\hline LVEDV(Iv) & $109.4 \pm 24.04$ & $110.7 \pm 11$ & 1.51 & 0.134 \\
Mean \pm SD & $35-159$ & $60-130$ & & \\
Range & & &
\end{tabular}




\begin{tabular}{lcccc}
\hline Table (1) Continue & & & & \\
\hline LVESV(lv) & $40.3 \pm 9.6$ & $41.8 \pm 5.6$ & 1.15 & 0.314 \\
Mean \pm SD & $17.9-53$ & $18-55$ & & \\
Range & & & & \\
EF $(\%)$ & $67.3 \pm 3.34$ & $67.56 \pm 3.77$ & 0.25 & 0.801 \\
Mean \pm SD & $60-73$ & $59-73$ & & \\
Range & & & \\
\hline
\end{tabular}

Table (2) Comparison between the two studied groups as regard tissue strain parameters.

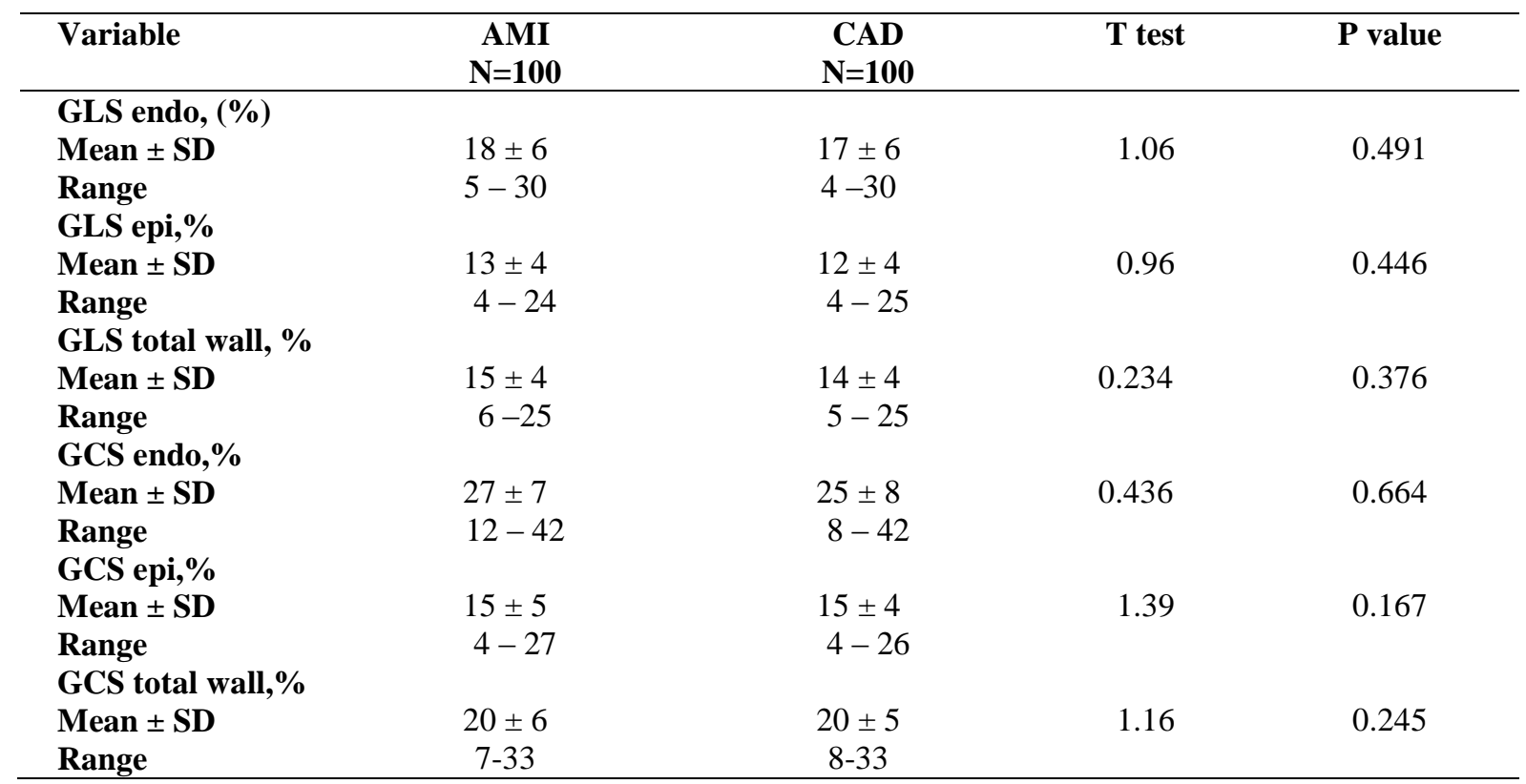

Table (3) Conventional echocardiography parameters with and without cardiac event in patients with first acute myocardial infarction.

\begin{tabular}{lcccc}
\hline Variable & $\begin{array}{c}\text { No cardiac event } \\
\mathbf{N}=\mathbf{7 5}\end{array}$ & $\begin{array}{c}\text { Cardiac event } \\
\mathbf{N = 2 5}\end{array}$ & T test & P value \\
\hline LVEDV(lv) & $115.4 \pm 22.04$ & $110.7 \pm 11$ & 4.51 & $<0.001$ \\
Mean \pm SD & $65-159$ & $35-133$ & & $(\mathrm{HS})$ \\
Range & & & & $<0.041$ \\
LVESV(lv) & $44.3 \pm 5.9$ & $39.8 \pm 5.4$ & 3.25 & $(\mathrm{~S})$ \\
Mean \pm SD & $24-53$ & $17.9-50$ & & $<0.001$ \\
Range & & & 6.25 & $(\mathrm{HS})$ \\
EF $(\%)$ & $69.3 \pm 4.39$ & $64.51 \pm 4.17$ & & \\
Mean \pm SD & $63-73$ & $60-73$ & & \\
Range & & & & \\
\hline
\end{tabular}

Table (4) Strain parameters with and without cardiac event in patients with first acute myocardial infarction.

\begin{tabular}{lcccc}
\hline Variable & $\begin{array}{c}\text { No cardiac event } \\
\mathbf{N = 7 5}\end{array}$ & $\begin{array}{c}\text { Cardiac event } \\
\mathbf{N = 2 5}\end{array}$ & T test & P value \\
\hline GLS endo, (\%) & $19 \pm 5$ & $16 \pm 4$ & 3.06 & 0.031 \\
Mean \pm SD & $6-30$ & $5-30$ & & $(\mathrm{~S})$ \\
Range & & & 6.1 & $<0.001$ \\
GLS epi, \% & $15 \pm 3$ & $11 \pm 3$ & & $(\mathrm{HS})$ \\
Mean \pm SD & $7-24$ & $4-24$ & & $<0.001$ \\
Range & $16 \pm 4$ & $13 \pm 3$ & 0.234 & $(\mathrm{HS})$ \\
GLS total wall, \% & $8-25$ & $6-20$ & & \\
Mean \pm SD & & & & \\
Range & & & & \\
\hline
\end{tabular}




\begin{tabular}{lcccc}
\hline Table (4) Continue & & & & \\
\hline Mean \pm SD & $28 \pm 7$ & $24 \pm 7$ & 0.436 & $<0.001$ \\
Range & $16-42$ & $12-40$ & & (HS) \\
GCS epi, \%: & & & 3.39 & 0.008 \\
Mean \pm SD & $16 \pm 3$ & $14 \pm 3$ & & $($ S) \\
Range & $7-27$ & $4-25$ & & 0.021 \\
GCS total wall, \% & & & 2.11 & (S) \\
Mean \pm SD & $21 \pm 4$ & $19 \pm 4$ & & \\
Range & $8-33$ & $7-30$ & & \\
\hline
\end{tabular}

Table (5) Conventional echocardiography parameters with and without cardiac event in patients with Coronary artery disease.

\begin{tabular}{|c|c|c|c|c|}
\hline Variable & $\begin{array}{c}\text { No cardiac event } \\
\quad \mathrm{N}=60\end{array}$ & $\begin{array}{c}\text { Cardiac event } \\
\mathrm{N}=\mathbf{4 0}\end{array}$ & T test & $P$ value \\
\hline \multicolumn{5}{|l|}{ LVEDV(lv) } \\
\hline Mean \pm SD & $111.4 \pm 10.14$ & $109.7 \pm 10$ & 4.59 & 0.041 \\
\hline Range & 70-130 & $60-120$ & & $(\mathrm{~S})$ \\
\hline \multicolumn{5}{|l|}{ LVESV(Iv) } \\
\hline Mean \pm SD & $42.3 \pm 5.1$ & $39.8 \pm 5.6$ & 3.15 & 0.046 \\
\hline Range & $20-53$ & $18-55$ & & $(\mathrm{~S})$ \\
\hline \multicolumn{5}{|l|}{ EF (\%) } \\
\hline Mean \pm SD & $69.3 \pm 3.31$ & $64.56 \pm 3.71$ & 5.15 & 0.009 \\
\hline Range & $61-73$ & $59-70$ & & $(\mathrm{~S})$ \\
\hline
\end{tabular}

Table (6) Strain parameters with and without cardiac event in patients with Coronary artery disease.

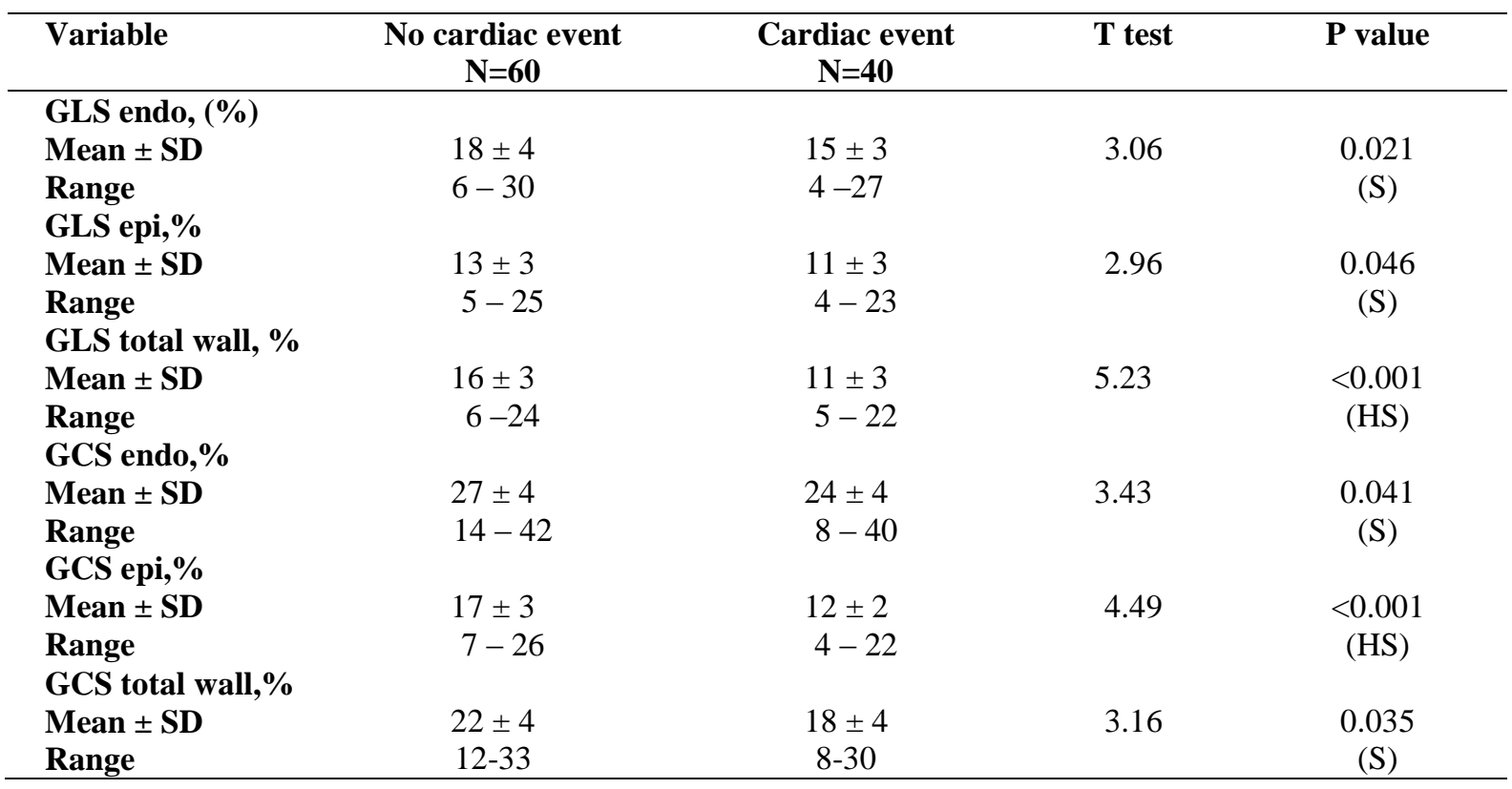

\section{Discussion}

Recently, clinical studies have investigated the diagnostic value of 2D strain during DSE for inducible ischemia detection in patients with suspected CAD, with special emphasis on the myocardial longitudinal strain reserve (LSR) with speckle-tracking echo, defined as the reserve between peak stress and rest during DSE, which reflects the ability of the myocardium to improve its function during stress testing [7].

In the current study there is no significant difference between the studied groups as regard LVESV, also there is no significant difference between the studied groups as regard LVEDV and EF.
Our results were in agreement with study of [8] as they reported that there was no significant difference between the studied groups as regard EF.

However, J.Liszka [9] reported that there was significant difference between the studied groups as regard LVEDV, LVESV and EF.

The two dimensional-speckle tracking echocardiography (2D-STE) has been used to assess the global and regional myocardial functions of the left ventricle (LV), since it automatically recognizes two dimensional myocardial speckles to track the myocardial trajectory and quantitatively analyzes the longitudinal, 
radial and circumferential myocardial strains of the left ventricle [10].

The present study showed that there was no statistically significant difference between the two studied groups as regard strain parameters (GCS \& GLS).

Our results were supported by study of [11] as they reported that there was no statistically significant difference between the two studied groups as regard strain parameters GCS.

However, [12] revealed that there was a statistically significant difference between the study groups regarding stress GLS.

The current study showed that as regard patients with acute myocardial infarction, there is high significant difference between cases with cardiac event and those without as regard LVEDV (lv) and EF\%, also there is significant difference between cases with cardiac event and those without as regard LVESV (lv). There is high significant difference between cases with cardiac event and those without as regard GLS epi,\%, GLS total wall, $\%$ and GCS endo, $\%$, also there is significant difference between cases with cardiac event and those without as regard GLS endo, (\%), GCS epi,\% and GCS total wall,\%.

Our results were supported by study of [13] as they revealed that among the strain variables examined, GLS $(\mathrm{P}=0.045)$ and GCS $(\mathrm{P}=0.004)$ endocardial strain parameters showed the greatest differences in those with and without cardiac event AMI group.

Many domestic and international studies conducted by $[14,15]$ have found that GLS could accurately detect the MIS (Myocardial infarction size) and had a higher diagnostic value than LVEF and WMSI [16] applied 3DSTE to evaluate the MIS and found that patients with a bigger MIS had lower values of 3D strain parameters. These studies also showed that GCS, GLS, GRS, WMSI (wall motion score index), and LVEF were all well correlated with the MIS detected by the magnetic resonance imaging (MRI).

In patients after AMI, the majority of studies have examined the prognostic value of GLS as a parameter of longitudinal deformation performance [3]. Similar to the AMI setting, 2D STE has also been recently utilized to provide prognostic information in CAD patients [17]. These studies never directly compared these 2 patient types.

[18] observed that after adjusting for potential confounders, midwall, endocardial, and epicardial GLS were significant predictors of fatal and nonfatal cardiovascular events.

[19] found that the averaged strain obtained in all LV segments (global longitudinal strain) is a reliable predictor of LVR $(\geq 15 \%$ increase in LV EDV at 6 months after AMI) with a sensitivity of $84.8 \%$ and specificity of $87.8 \%$. [20] related 4-chamber and 2chamber global peak longitudinal strain and parasternal short-axis global circumferential strain to the LVR at 20month follow-up. Although both types of strain were associated with clinical outcomes, only circumferential analysis was found to be predictive of LVR $(\geq 15 \%$ increase in LV ESV assessed at 20-month follow-up)..
[21] reported that impaired LV torsion may help predict LVR assessed 6 months after the AMI and defined as $\geq 15 \%$ or $\geq 20 \%$ increase in LV EDV.

In the study in our hands, as regard patients with coronary artery disease, there is significant difference between cases with cardiac event and those without as regard LVEDV (lv), EF\%, and LVESV (lv). There is high significant difference between cases with cardiac event and those without as regard GLS epi, \% and GLS total wall, also there is significant difference between cases with cardiac event and those without as regard GLS endo, (\%), GLS epi, \%, GCS endo and GCS total wall, \%.

Our results were supported by study of [13] as they reported that a more pronounced separation between patients with and without a cardiac event was observed in CAD patients. GCS \& GLS parameters attained statistical significance in patients with and without a cardiac event ( $\mathrm{P}<0.001$ and $\mathrm{P}=0.011$, respectively).

In recent studies carried out by [22] reported that longitudinal strain has been recognized as a sensitive parameter in the detecting $\mathrm{CAD}$ at rest even in the presence of normal LV wall motion in patients with severe CAD.

[23] who found a significant negative correlation between longitudinal strain and severity of CAD as assessed by coronary angiography. This agrees with the fact that longitudinal mechanics predominate in the ischemia-vulnerable subendocardium as subendocardial myocardial fibers are mainly longitudinal oriented and abnormalities of myocardial deformation in the longitudinal axis are seen in the development of many pathophysiologic states, including $\mathrm{CAD}$ and myocardial infarction [24].

\section{Conclusion}

Results from the present study demonstrate that layerspecific 2D STE may be considered as a sensitive tool that is capable of predicting cardiac events in CAD patients, which may not be detected using conventional echocardiographic parameters. In contrast, 2D STE fails to increase the predictive power in patients with AMI, probably due to acute myocardial edema and myocardial stunning. On this basis, we believe that extensive echocardiography does not offer any significant benefit for AMI patients in the acute setting.

\section{References}

[1] S.A. Hunt, W.T. Abraham, M.H. Chin, American College of Cardiology; American Heart Association Task Force on Practice Guidelines; American College of Chest Physicians; International Society for Heart and Lung Transplantation; Heart Rhythm Society. ACC/AHA 2005 guideline update for the diagnosis and management of chronic heart failure in the adult: a report of the American College of Cardiology/American Heart Association Task Force on Practice Guidelines (Writing Committee to Update the 2001 Guidelines for the Evaluation and Management of Heart Failure): developed in collaboration with the American College of Chest 
Physicians and the International Society for Heart and Lung Transplantation: endorsed by the Heart Rhythm Society. Circulation;Vol.112, pp. 235154,2005 .

[2] J.E. Møller, G.S. Hillis, Oh JK, Wall motion score index and ejection fraction for risk stratification after acute myocardial infarction;Vol.151, pp.425419,2006.

[3] M. Ersbøll, N. Valeur, U.M. Mogensen,Prediction of all-cause mortality and heart failure admissions from global left ventricular longitudinal strain in patients with acute myocardial infarction and preserved left ventricular ejection fraction. J Am Coll Cardiol; Vol. 61, pp. 2373-2365,2013.

[4] E. Altiok, S. Tiemann, M. Becker, Myocardial deformation imaging by two-dimensional speckletracking echocardiography for prediction of global and segmental functional changes after acute myocardial infarction: a comparison with late gadolinium enhancement cardiac magnetic resonance. J Am Soc Echocardiogr;Vol. 27, pp.257-249, 2014.

[5] T. Biering-Sørensen, S. Hoffmann, R. Mogelvang, Myocardial strain analysis by 2-dimensional speckle tracking echocardiography improves diagnostics of coronary artery stenosis in stable angina pectoris. Circ Cardiovasc Imaging; Vol.7, PP. 65- 58,2014.

[6] S. Windecker, P. Kolh, F. Alfonso, ESC/EACTS Guidelines on myocardial revascularization: the Task Force on Myocardial Revascularization of the European Society of Cardiology (ESC) and the European Association for Cardio-Thoracic Surgery (EACTS)Developed with the special contribution of the European Association of Percutaneous Cardiovascular Interventions (EAPCI);Vol.35, PP.2619-2541,2014.

[7] T. Cognet, P.L. Vervueren, L. Dercle, New concept of myocardial longitudinal strain reserve assessed by a dipyridamole infusion using 2D-strain echocardiography: the impact of diabetes and age, and the prognostic value. Cardiovasc Diabetol;Vol.12, PP.84-95,2013.

[8] J. Schroeder, S.Hamada, N.Gründlinger, T.Rubeau, Myocardial deformation by strain echocardiography identifies patients with acute coronary syndrome and non-diagnostic ECG presenting in a chest pain unit: a prospective study of diagnostic accuracy. Clinical Research in Cardiology; Vol.105, PP.256248,2016.

[9] J.Liszka, M.Haberka, , Z.Tabor, Two-dimensional speckle-tracking echocardiography assessment of left ventricular remodeling in patients after myocardial infarction and primary reperfusion. Archives of medical science;Vol.10, PP. 9110,2014 .

[10] M. Leitman, P. Lysyansky, S. Sidenko, Twodimensional strain - a novel software for real-time quantitative echocardiographic assessment of myocardial function. J Am Soc Echocardiogr;Vol.17, PP.1029-1021,2004.

[11] M. Xie, , J.Yin, Assessment of the left ventricular systolic function in multi-vessel coronary artery disease with normal wall motion by twodimensional speckle tracking echocardiography. Eur Rev Med Pharmacol Sc;Vol. 19, PP.39343928,2015.

[12] A. A.Aziz, R. D.AbouShokka \& M. M. Abdelsamie, Myocardial longitudinal strain reserve as a marker of severity of coronary artery disease during dobutamine stress echocardiography. Journal of Medicine in Scientific Research;Vol.3, PP.6474,2020 .

[13] J.Scharrenbroich, , S.Hamada, , A.Keszei, Use of two-dimensional speckle tracking echocardiography to predict cardiac events: Comparison of patients with acute myocardial infarction and chronic coronary artery disease. Clinical Cardiolog;Vol.41, PP.118-111,2018.

[14] B. Sjoli, S. Orn, B. Grenne, T. Vartdal, Comparison of left ventricular ejection fraction and left ventricular global strain as determinants of infarct size in patients with acute myocardial infarction. J Am Soc Echocardiogr;Vol.22, PP.1238-1232,2009.

[15] A. Thorstensen, H. Dalen, P. Hala, G. Kiss, Threedimensional echocardiography in the evaluation of global and regional function in patients with recent myocardial infarction: a comparison with magnetic resonance imaging. Echocardiography;Vol.30, PP.692-628,2013.

[16] W. Zhu, W. Liu, Y. Tong, Three-dimensional speckle tracking echocardiography for the evaluation of the infarct size and segmental transmural involvement in patients with acute myocardial infarction. Echocardiography; Vol.31, PP. 66- 58,2014.

[17] T. Biering-Sørensen, S. Hoffmann, R. Mogelvang, Myocardial strain analysis by 2-dimensional speckle tracking echocardiography improves diagnostics of coronary artery stenosis in stable angina pectoris. Circ Cardiovasc Imaging; Vol.7, PP. 65- 58,2014.

[18] T. Kuznetsova, N. Cauwenberghs, J. Knez, Additive prognostic value of left ventricular systolic dysfunction in a population-based cohort. Circ Cardiovasc Imaging; Vol.9, PP. 46-61,2016.

[19] A. D'Andrea, R. Cocchia, P. Caso, Global longitudinal speckle-tracking strain is predictive of left ventricular remodeling after coronary angioplasty in patients with recent non-ST elevation myocardial infarction. Int $\mathbf{J}$ Cardiol;Vol. 91, PP.185-153,2011.

[20] C. Hung, A. Verma, H. Uno, Longitudinal and circumferential strain rate, left ventricular remodeling, and prognosis after myocardial infarction. J Am Coll Cardiol;Vol.56, PP. 2218,2010 . 
[21] G. Nucifora, N.A. Marsan, M. Bertini, Reduced left ventricular torsion early after myocardial infarction is related to left ventricular remodeling. Circ Cardiovasc Imaging;Vol.34, PP. 42-33,2010.

[22] M.P. Mahjoob, S. AlipourParsa, A. Mazarei, Rest 2D speckle tracking echocardiography may be a sensitive but nonspecific test for detection of significant coronary artery disease. Acta Biomed;Vol. 88, PP. 461-457,2018.
[23] A. Vrettos, D. Dawson, C. Grigoratos, Correlation between global longitudinal peak systolic strain and coronary artery disease severity as assessed by the angiographically derived SYNTAX score. Echo Res Pract;Vol.3, PP. 34- 29,2016.

[24] N. Gaibazzi, F. Pigazzani, C. Reverberi, Rest global longitudinal 2D strain to detect coronary artery disease in patients undergoing stress echocardiography: a comparison with wall-motion and coronary flow reserve responses. Echo Res Pract; Vol.1, PP. 70-61,2014. 